\title{
A Modulation Strategy for Improving Output Performance of Matrix Converter
}

\author{
Yaguang Ma, Xinghe Ma, Peiru Li, and Xin Ren
}

\begin{abstract}
Based on the traditional principle of two-line voltage synthesis, a modulation strategy is proposed to improve the output performance of matrix converter by changing the switching mode. According to the switching mode of the new strategy, under the premise that the input current and output voltage are sinusoidal, the modulation time of each switch of the matrix converter in one cycle is derived from the relationship between input and output current and voltage. Under the same conditions, the proposed modulation strategy is compared with the traditional two-line voltage synthesis strategy, and the corresponding simulation data and some experimental waveforms are given. Based on the research of MATLAB software simulation, the control strategy of the DSPACE hardware real-time simulation platform is experimentally studied. The simulation and experimental results verify the correctness of the modulation strategy.
\end{abstract}

Index Terms-Matrix converter, modulation strategy, output performance, voltage harmonics.

\section{INTRODUCTION}

$\mathrm{T}$ HE topology of the three-phase input to the three-phase output matrix converter (MC) is shown in Fig. 1. Threephase output can be connected to any phase input through nine bidirectional switches [1]-[3]. The desired three-phase output voltage and frequency can be obtained by controlling nine bidirectional switches with certain strategy [4], [5]. Compared with the traditional AC-AC converter, $\mathrm{MC}$ has many advantages, such as bidirectional energy transmission [6], sinusoidal input and output [7], adjustable power factor etc [8], [9]. According to the different control objectives, the modulation strategies of MC can be divided into current control method and voltage control method. Among them, the two-voltage synthesis method [10]-[13] and the space vector method [14], [15] are widely used for voltage control method. In two-line voltage modulation method, the power factor is larger when

Manuscript received July 20, 2019. This work was partly financially supported through grants from the National Key Research and Development Plan of China (No. 2016YFC0600906), and the Natural Science Foundation of Henan Province, China (No. 182300410280).

Y. Ma, X. Ma, and X. Ren are with the School of Electrical Engineering and Automation, Henan Polytechnic University, Jiaozuo, 454150, China (e-mail: myghpu@163.com).

P. Li is with State Grid Henan Power Company Zhumadian Power Supply Company, Zhumadian 463000, Henan, China (e-mail: hexueshu@163.com).

Digital Object Identifier 10.24295/CPSSTPEA.2019.00024

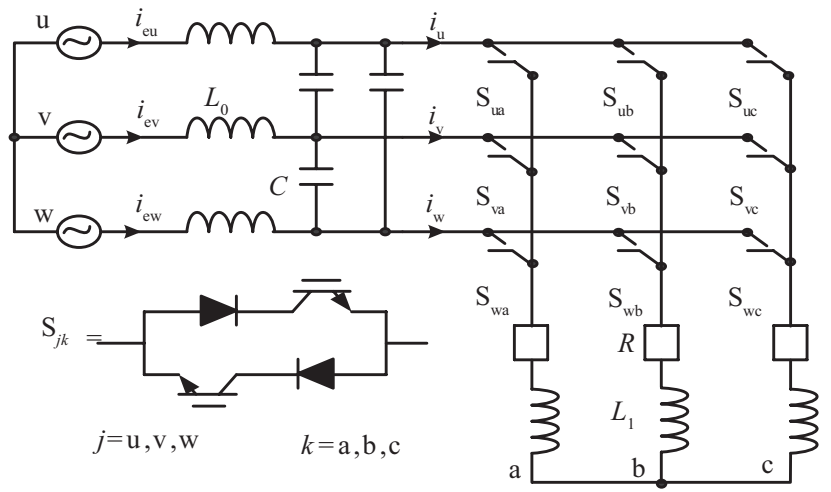

Fig. 1. Topology of matrix converter.

two maximum input line voltages are used to synthesize output line voltages, but the harmonic content is larger [16], [17]. In addition, when the output voltage is low, due to the existence of a large number of narrow pulses, the harmonics in the output line voltage are greatly increased, and the waveform is severely distorted [18]. Aiming at the disadvantage of serious distortion of output voltage waveform at low voltage output, literature [19] uses two smaller input line voltages to synthesize the output voltage, and the low-voltage output performance is improved, but its maximum voltage gain is only 0.5 . To overcome the shortcomings of traditional two-line voltage modulation strategy, this paper proposes a modulation strategy to improve the output performance of MC. The new strategy not only improves the output performance of the converter, but also achieves a maximum voltage gain of 0.866 . Its main features are: in a sampling period, when the output voltage is high, the maximum output line voltage is formed by three input line voltage pulse waveform, and the other two-phase output line voltages are formed by non-maximum two-input line voltage pulse waves and zero voltage. Under the same conditions, the number of narrow pulses is less than that of the traditional twovoltage synthesis modulation strategy. When the output voltage is low, the three-output line voltage is formed by the nonmaximum two-input line voltage pulse waves and zero voltage. Compared with the traditional two-line voltage synthesis modulation strategy, the number of narrow pulses is greatly reduced, the harmonic of output voltage is greatly suppressed, and the output performance of the converter is improved. The correctness of the new modulation strategy is verified by simulation and experiment. 


\section{Principle of New Modulation Strategy}

\section{A. Proposal of New Modulation Strategy}

For ease of analysis, the following equations and variables are defined:

Maximum, middle and minimum input phase voltage:

$$
\left\{\begin{array}{l}
e_{\text {max }}=\max \left(e_{\mathrm{u}}, e_{\mathrm{v}}, e_{\mathrm{w}}\right) \\
e_{\text {mid }}=\operatorname{mid}\left(e_{\mathrm{u}}, e_{\mathrm{v}}, e_{\mathrm{w}}\right) \\
e_{\text {min }}=\min \left(e_{\mathrm{u}}, e_{\mathrm{v}}, e_{\mathrm{w}}\right)
\end{array}\right.
$$

Corresponding input phase voltage $e_{\max }, e_{\operatorname{mid}}, e_{\min }$, the current is $i_{\mathrm{i}(\max )}, i_{\mathrm{i}(\operatorname{mid})}, i_{\mathrm{i}(\min )}$.

Maximum, middle and minimum input line voltage:

$$
\left\{\begin{aligned}
\Delta e_{\text {max }} & =e_{\text {max }}-e_{\text {min }} \\
\Delta e_{\text {mid }} & =\left\{\begin{array}{l}
e_{\text {max }}-e_{\text {mid }} ; e_{\text {base }}=e_{\text {max }} \\
e_{\text {mid }}-e_{\text {min }} ; e_{\text {base }}=e_{\text {min }}
\end{array}\right\} \\
\Delta e_{\text {min }} & =\left\{\begin{array}{l}
e_{\text {mid }}-e_{\text {min }} ; e_{\text {base }}=e_{\text {max }} \\
e_{\text {max }}-e_{\text {mid }} ; e_{\text {base }}=e_{\text {min }}
\end{array}\right\}
\end{aligned}\right.
$$

$e_{\text {base }}$ is the phase voltage with the maximum absolute value in the input phase voltage.

Maximum, middle and minimum output phase voltage:

$$
\left\{\begin{array}{l}
v_{\text {max }}=\max \left(v_{\mathrm{a}}, v_{\mathrm{b}}, v_{\mathrm{c}}\right) \\
v_{\text {mid }}=\operatorname{mid}\left(v_{\mathrm{a}}, v_{\mathrm{b}}, v_{\mathrm{c}}\right) \\
v_{\text {min }}=\min \left(v_{\mathrm{a}}, v_{\mathrm{b}}, v_{\mathrm{c}}\right)
\end{array}\right.
$$

Corresponding output phase voltage $v_{\max }, v_{\operatorname{mid}}, v_{\min }$, the current is $i_{\mathrm{o}(\max )}, i_{\mathrm{o}(\operatorname{mid})}, i_{\mathrm{o}(\min )}$.

Maximum, middle and minimum output phase voltage:

$$
\left\{\begin{array}{l}
\Delta v_{\text {max }}=v_{\text {max }}-v_{\text {min }} \\
\Delta v_{\text {mid }}=\left\{\begin{array}{l}
v_{\text {max }}-v_{\text {mid }} ; v_{\text {base }}=v_{\text {max }} \\
v_{\text {mid }}-v_{\text {min }} ; v_{\text {base }}=v_{\text {min }}
\end{array}\right\} \\
\Delta v_{\text {mid }}=\left\{\begin{array}{l}
v_{\text {mid }}-v_{\text {min }} ; v_{\text {base }}=v_{\text {max }} \\
v_{\text {max }}-v_{\text {mid }} ; v_{\text {base }}=v_{\text {min }}
\end{array}\right\}
\end{array}\right.
$$

$v_{\text {base }}$ is the phase voltage with the maximum absolute value in the output phase voltage.

The modulation time of 9 switches of matrix converter and the corresponding modulation coefficient should satisfy the following relationship:

$$
\left\{\begin{array}{l}
d_{j k}=T_{j k} / T_{\mathrm{S}} \\
d_{\mathrm{u} k}+d_{\mathrm{v} k}+d_{\mathrm{w} k}=1,
\end{array} j=\mathrm{u}, \mathrm{v}, \mathrm{w}, k=\mathrm{a}, \mathrm{b}, \mathrm{c}\right.
$$

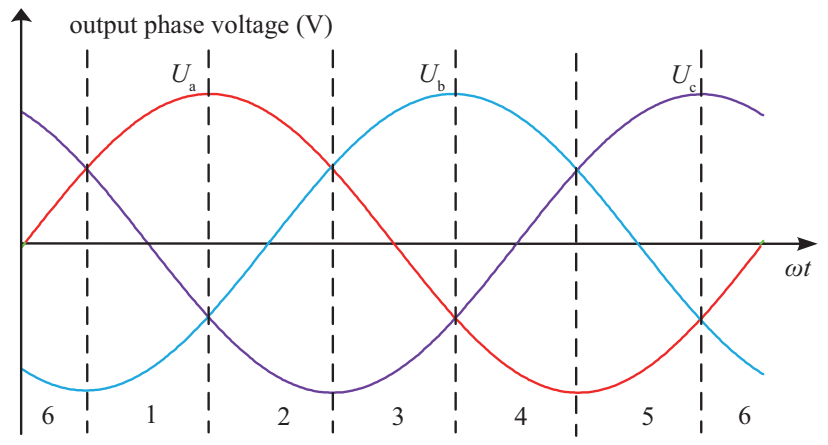

Fig. 2. The sector partition of output voltages.

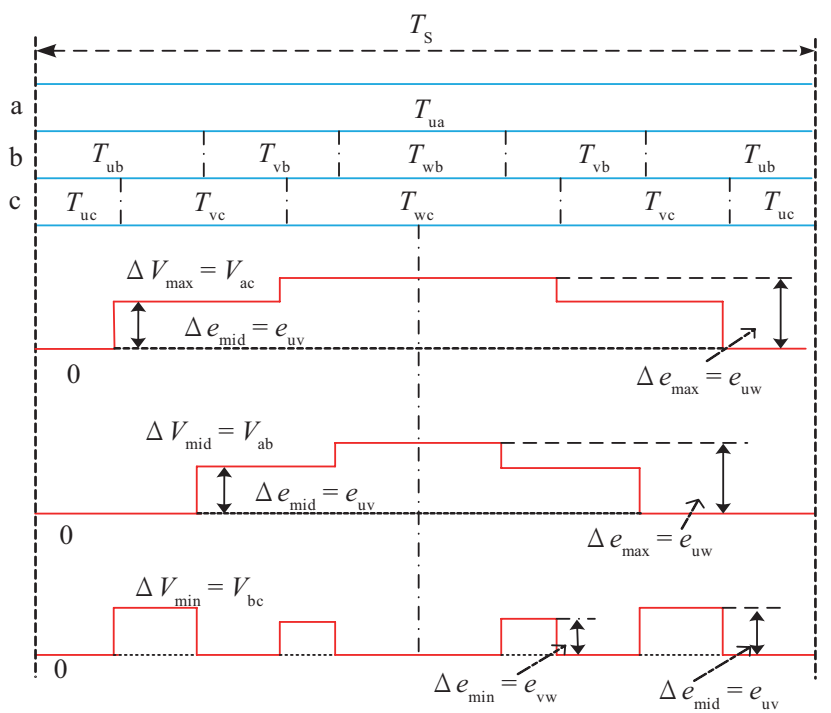

Fig. 3. Switching patterns and output voltage waveforms in conventional twoline voltage synthesis.

$T_{\mathrm{S}}$ is the sampling period.

In order to facilitate the understanding of the principle and characteristics of the new modulation strategy, a comparative analysis is made with the traditional two-line voltage synthesis method. The output reference phase voltage and the input phase voltage are divided into sections as shown in Fig. 2. Taking $i_{\mathrm{i}(\mathrm{mid})}<0$, the input phase voltage and the output reference phase voltage are both in 2 sections as examples. In the traditional two-line voltage synthesis modulation strategy, the switching mode of synthesizing three output line voltage and the output line voltage waveform are shown in Fig. 3. As can be seen from Fig. 3, the maximum phase of the output phase voltage is always connected with the maximum phase of the input phase voltage during a sampling period, and the six switches connected with the other two phases are pulse width modulated. The large and medium phases of the output line voltage are synthesized by the large and medium phases of the input line voltage. Thus, there are two zero voltage and maximum input line voltage sections in the line voltage waveform, and there are larger harmonics, and only the small phases of the output voltage are synthesized by the medium and small phases of the input line voltage [20], [21]. In 


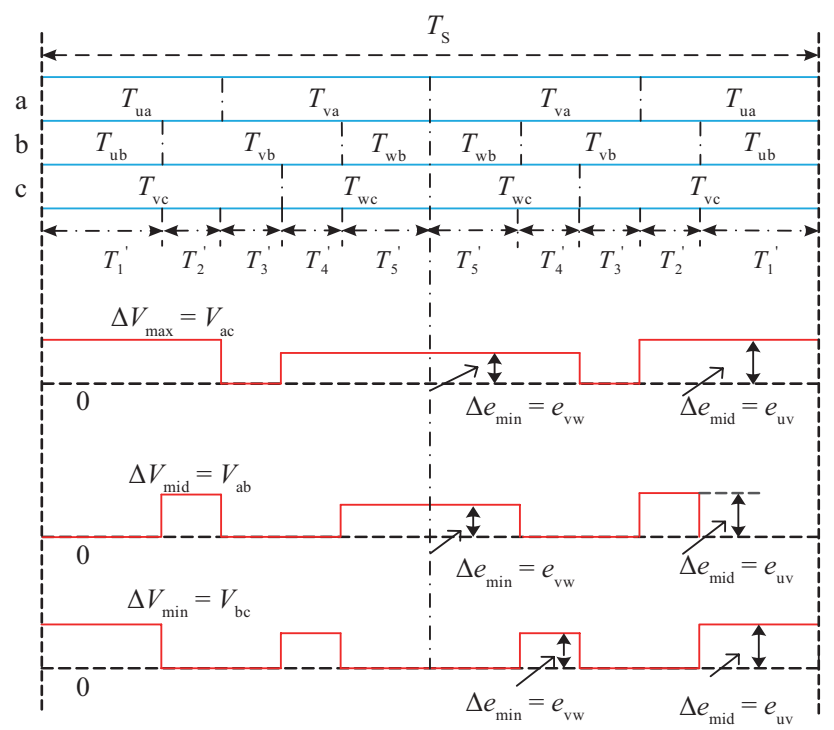

(a)

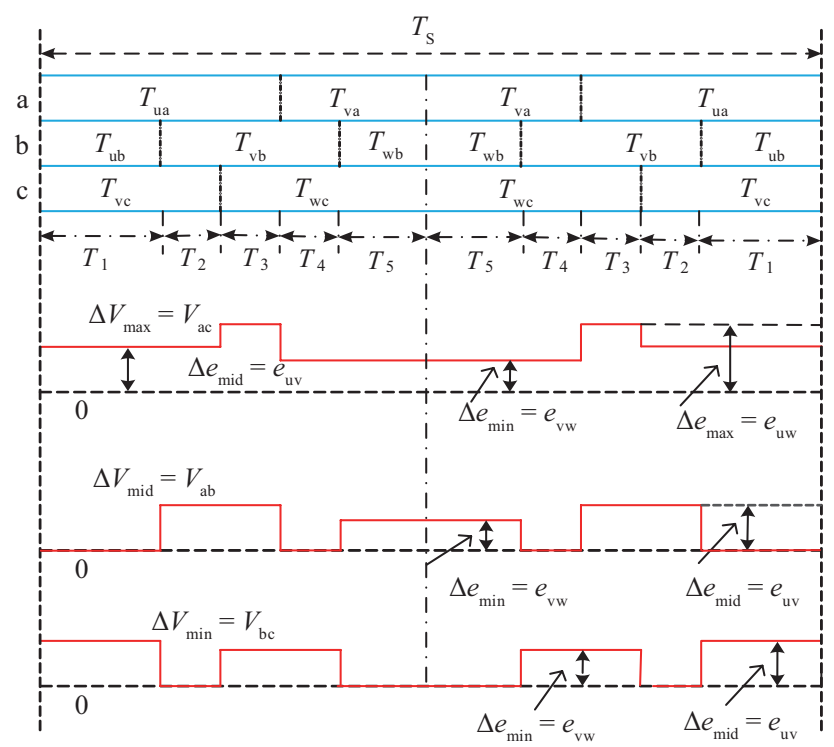

(b)

Fig. 4. Switching mode and output voltage waveform of the new modulation strategy. (a) Low voltage output. (b) High voltage output.

addition, when the output voltage is low, due to the existence of a large number of narrow pulses, the harmonics in the output line voltage are greatly increased, and the waveform is seriously distorted, which affects the output performance of matrix converter.

Fig. 4 shows the switching mode and output voltage waveform of the new modulation strategy. In a sampling period, the maximum value of reference output phase voltage is only connected with the maximum and middle value of input phase voltage, the minimum value of reference output phase voltage is only connected with the middle and minimum value of input voltage, and the middle value of reference output phase voltage is connected with the maximum, middle and minimum value of input phase voltage [22]. It can be seen that the maximum output line voltage is composed of three-input line voltage pulse wave at high output voltage, while the other two-phase output voltage is composed of non-maximum two-input line voltage pulse and zero-voltage[23]. Compared with the traditional two-voltage synthesis strategy, the output voltage harmonics strategy is reduced. When the output voltage is low, the three-output line voltage is composed of two nonmaximum input line voltage pulses and zero voltage. Under the same low-voltage output, the number of narrow pulses is greatly reduced compared with the traditional two-line voltage synthesis strategy, and the output performance of matrix converter is greatly improved.

\section{B. Modulation Strategy Implementation}

In order to simultaneously satisfy the sinusoidal input current and output voltage, the modulation time of each switch of matrix converter in a period can be obtained from the relationship between input current and output voltage. Taking the switching mode of Fig. 4(b) as an example, the modulation time of each switch of the matrix converter in one cycle is derived. From the switching mode of Fig. 4(b), the equation between three-phase input current and output current, output line voltage and input line voltage can be obtained as follows:

$$
\begin{aligned}
& \left\{\begin{array}{l}
i_{\mathrm{i}(\max )}=\left(2 T_{1}+2 T_{2}+2 T_{3}\right) i_{\mathrm{o}(\max )}+2 T_{1} i_{\mathrm{o}(\operatorname{mid})} \\
i_{\mathrm{i}(\operatorname{mid})}=\left(-2 T_{1}-2 T_{2}+2 T_{4}+2 T_{5}\right) i_{\mathrm{o}(\max )}+\left(-2 T_{1}+2 T_{3}+2 T_{4}\right) i_{\mathrm{o}(\operatorname{mid})} \\
i_{\mathrm{i}(\min )}=\left(-2 T_{3}-2 T_{4}-2 T_{5}\right) i_{\mathrm{o}(\max )}+\left(-2 T_{3}-2 T_{4}\right) \cdot i_{o(\operatorname{mid})}
\end{array}\right. \\
& \left\{\begin{array}{l}
\Delta v_{\text {max }}=\left[\left(2 T_{1}+2 T_{2}\right) \Delta e_{\text {mid }}+2 T_{3} \Delta e_{\text {max }}+\left(2 T_{4}+2 T_{5}\right) \Delta e_{\text {min }}\right] / T_{\mathrm{S}} \\
\Delta v_{\text {mid }}=\left[\left(2 T_{2}+2 T_{3}\right) \Delta e_{\text {mid }}+2 T_{5} \Delta e_{\text {min }}\right] / T_{\mathrm{S}} \\
\Delta v_{\text {min }}=\left[2 T_{1} \Delta e_{\text {mid }}+\left(2 T_{3}+2 T_{4}\right) \Delta e_{\text {min }}\right] / T_{\mathrm{S}}
\end{array}\right.
\end{aligned}
$$

To ensure that the input current is a sinusoidal quantity, formula (6) has:

$$
\frac{e_{\max }}{e_{\min }}=\frac{i_{\mathrm{i}(\max )}}{i_{\mathrm{i}(\min )}}=\frac{2 T_{1}+2 T_{2}+2 T_{3}}{2 T_{3}+2 T_{4}+2 T_{5}}=-\frac{2 T_{1}}{2 T_{3}+2 T_{4}}
$$

According to formulas (8):

$$
2 T_{3}+2 T_{4}=\frac{2 T_{1} \cdot e_{\min }}{e_{\max }}
$$

Bringing the formula (9) into the third formula of the formula (7)

$$
2 T_{1}=\frac{e_{\text {max }} \cdot \Delta v_{\text {min }} \cdot T_{\mathrm{S}}}{e_{\text {max }}^{2}+e_{\text {mid }}^{2}+e^{2}{ }_{\text {min }}}
$$


From equations (9) and (10):

$$
2 T_{3}+2 T_{4}=-\frac{e_{\text {min }} \cdot \Delta \nu_{\text {min }} \cdot T_{\mathrm{S}}}{e_{\text {max }}^{2}+e_{\text {mid }}^{2}+e_{\text {min }}^{2}}
$$

According to formulas (8), (10), (11):

$$
T_{2}+2 T_{3}=\frac{2 T_{5} \cdot e_{\max }}{e_{\min }}
$$

From the second formula of equations (12) and (7):

$$
\begin{gathered}
2 T_{5}=-\frac{e_{\text {min }} \cdot \Delta v_{\text {mid }} \cdot T_{\mathrm{S}}}{e_{\text {max }}^{2}+e_{\text {mid }}^{2}+e_{\text {min }}^{2}} \\
2 T_{2}+2 T_{3}=-\frac{e_{\text {max }} \cdot \Delta v_{\text {mid }} \cdot T_{\mathrm{S}}}{e_{\text {max }}^{2}+e_{\text {mid }}^{2}+e_{\text {min }}^{2}}
\end{gathered}
$$

According to the switching mode diagram of Fig. 4(b), the modulation time of three switches connected with the output maximum phase voltage can be obtained as follows:

$$
\left\{\begin{array}{l}
T_{\text {ua }}=2 T_{1}+2 T_{2}+2 T_{3}=\frac{\Delta v_{\text {max }} \cdot e_{\text {max }} \cdot T_{\mathrm{S}}}{e_{\text {max }}^{2}+e_{\text {mid }}^{2}+e_{\text {min }}^{2}} \\
T_{\text {va }}=T_{\mathrm{S}}-T_{\text {ua }} \\
T_{\text {wa }}=0
\end{array}\right.
$$

The modulation time of the three switches connected to the output intermediate phase voltage is:

$$
\left\{\begin{array}{l}
T_{\mathrm{ub}}=2 T_{13}=\frac{\Delta v_{\text {min }} \cdot e_{\text {max }} \cdot T_{\mathrm{S}}}{e_{\text {max }}^{2}+e_{\text {mid }}^{2}+e_{\text {min }}^{2}} \\
T_{\mathrm{vb}}=T_{\mathrm{S}}-T_{\mathrm{ub}}-T_{\mathrm{wb}} \\
T_{\mathrm{wb}}=2 T_{5}=-\frac{\Delta v_{\text {mid }} \cdot e_{\text {min }} \cdot T_{\mathrm{S}}}{e_{\text {max }}^{2}+e_{\text {mid }}^{2}+e_{\text {min }}^{2}}
\end{array}\right.
$$

The modulation time of the three switches connected to the output minimum phase voltage is:

$$
\left\{\begin{array}{l}
T_{\mathrm{uc}}=0 \\
T_{\mathrm{vc}}=T_{\mathrm{S}}-T_{\mathrm{wc}} \\
T_{\mathrm{wc}}=2 T_{3}+2 T_{4}+2 T_{5}=-\frac{\Delta v_{\text {max }} \cdot e_{\text {min }} \cdot T_{\mathrm{S}}}{e_{\text {max }}^{2}+e_{\text {mid }}^{2}+e_{\text {min }}^{2}}
\end{array}\right.
$$

Similarly, the switching time of each switch of matrix converter can also be calculated according to the switching mode of Fig. 4(a), which is exactly the same as the switching mode of Fig. 4(b).

In the case of balanced input, because the maximum value of $\Delta v_{\max }$ is formula $\sqrt{3} V_{\text {om }}$, the maximum value of $e_{\max }$ is $E_{\mathrm{im}}$ in formula $e_{\max }^{2}+e_{\text {mid }}^{2}+e_{\min }^{2}=\frac{3}{2} \cdot E_{\mathrm{im}}^{2}$, and the expression of $T_{\text {ua }}$ by equation (15) has
TABLE I

SimUlation PARAMETERS

\begin{tabular}{lc}
\hline \multicolumn{1}{c}{ Parameter } & Numerical value \\
\hline Input filter inductor & $5 \mathrm{mH}$ \\
Filter capacitor & $6 \mu \mathrm{F}$ \\
Damping resistance & $15 \Omega$ \\
Three-phase symmetrical resistance load resistance & $15 \Omega$ \\
Inductance & $5 \mathrm{mH}$ \\
Valid value of input line voltage & $120 \mathrm{~V}$ \\
Fundamental frequency & $50 \mathrm{~Hz}$ \\
Sampling frequency & $5 \mathrm{kHz}$ \\
\hline \hline
\end{tabular}

$$
\begin{aligned}
0 \leqslant d_{\text {ua }} & =\frac{\Delta v_{\text {max }} \cdot e_{\text {max }}}{e_{\text {max }}^{2}+e_{\text {mid }}^{2}+e_{\text {min }}^{2}} \leqslant 1 \\
0 & \leqslant V_{\text {om }} \leqslant \frac{\sqrt{3}}{2} \cdot E_{\text {im }}
\end{aligned}
$$

It can be seen that the maximum voltage gain of the modulation strategy is $\sqrt{3} / 2$. The adjustment range of power factor angle $\varphi^{*}$ is: $-\pi / 6 \leqslant \varphi^{*} \leqslant \pi / 6$.

\section{SiMULATION AND EXPERIMENT}

To verify the feasibility and correctness of the proposed modulation strategy, a prototype experimental platform based on dSPACE as the main controller is designed. The dSPACE used in this experiment is a single processing system with DS1005 board as the core. The I/O boards are equipped with DS4002 (timing and digital I/O), DS5101 (digital waveform generator), DS2003 (A/D conversion) and DS2103 (D/A conversion). The characteristics and application of dSPACE real-time simulation platform are referred to references [24], [25]. Simulation and experimental parameters are shown in Table I.

In order to compare with the traditional two-line voltage synthesis modulation strategy, the data of the traditional twoline voltage synthesis and the proposed new modulation strategy under the same simulation parameters are given, as shown in Table II. The number of narrow pulses in the table is the count value in $0.02 \mathrm{~s}$. The simulation and experiment adopt three-step commutation based on output current, and the commutation is realized by staflow in MATLAB. The width of narrow pulses removed is 5 us. From the statistics in Table II, it can be seen that when the output voltage frequency is constant, the distortion rate and the number of narrow pulses of the new modulation strategy are obviously smaller than those of the traditional modulation strategy. Especially when the output voltage is low, the difference is obvious, which indicates the correctness of the theoretical analysis. It can be seen that the new modulation strategy reduces the harmonic of the output voltage and improves the output performance of the converter.

Fig. 5 show the experimental waveforms under traditional modulation strategies. Fig. 6 and Fig. 7 show the experimental waveforms new modulation strategy when the desired output voltage amplitude is $70 \mathrm{~V}, 32 \mathrm{~V}$, and the frequency is $80 \mathrm{~Hz}$. 
TABLE II

Modulation Strategy and Simulation Data

\begin{tabular}{|c|c|c|c|c|c|c|}
\hline \multirow{3}{*}{ Modulation strategy and output frequency } & \multicolumn{3}{|c|}{ Output phase voltage amplitude (32 V) } & \multicolumn{3}{|c|}{ Output phase voltage amplitude $(70 \mathrm{~V})$} \\
\hline & \multirow[b]{2}{*}{$\begin{array}{c}\text { Narrow } \\
\text { pulse } \\
\text { number }\end{array}$} & \multicolumn{2}{|c|}{ Output line current } & \multirow[b]{2}{*}{$\begin{array}{l}\text { Narrow } \\
\text { pulse } \\
\text { number }\end{array}$} & \multicolumn{2}{|c|}{ Output line current } \\
\hline & & $\begin{array}{l}\text { Fundamental } \\
\text { amplitude }\end{array}$ & $\begin{array}{c}\text { Distortion } \\
\text { rate } \%(\mathrm{THD})\end{array}$ & & $\begin{array}{c}\text { Fundamental } \\
\text { wave } \\
\text { amplitude }\end{array}$ & $\begin{array}{l}\text { Distortion } \\
\text { rate } \%(\mathrm{THD})\end{array}$ \\
\hline
\end{tabular}

\begin{tabular}{|c|c|c|c|c|c|c|}
\hline \multicolumn{7}{|c|}{ Traditional modulation strategy } \\
\hline $30 \mathrm{~Hz}$ & 320 & 2.055 & 6.29 & 260 & 4.594 & 6.25 \\
\hline $50 \mathrm{~Hz}$ & 268 & 2.048 & 11.97 & 108 & 4.565 & 5.96 \\
\hline $80 \mathrm{~Hz}$ & 340 & 2.041 & 10.43 & 270 & 4.535 & 3.14 \\
\hline $100 \mathrm{~Hz}$ & 312 & 2.027 & 11.94 & 268 & 4.494 & 5.03 \\
\hline $30 \mathrm{~Hz}$ & 65 & 2.063 & 4.88 & 30 & 4.570 & 2.21 \\
\hline $50 \mathrm{~Hz}$ & 53 & 2.061 & 5.95 & 19 & 4.571 & 2.68 \\
\hline $80 \mathrm{~Hz}$ & 68 & 2.050 & 4.5 & 30 & 4.532 & 1.91 \\
\hline $100 \mathrm{~Hz}$ & 62 & 2.038 & 3.55 & 28 & 4.491 & 3.19 \\
\hline
\end{tabular}

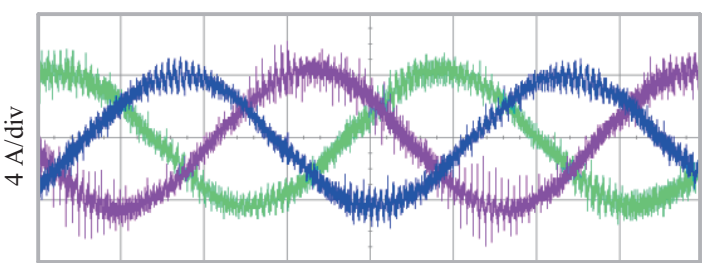

$2 \mathrm{~ms} / \mathrm{div}$

(a)

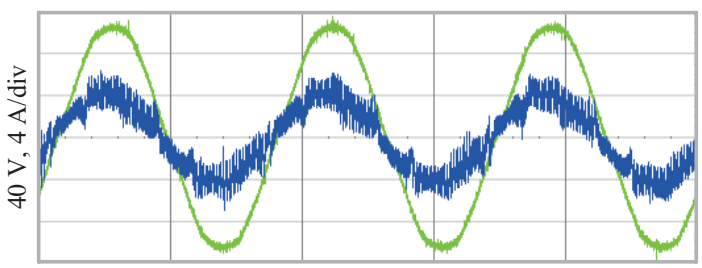

$12 \mathrm{~ms} / \mathrm{div}$

(b)

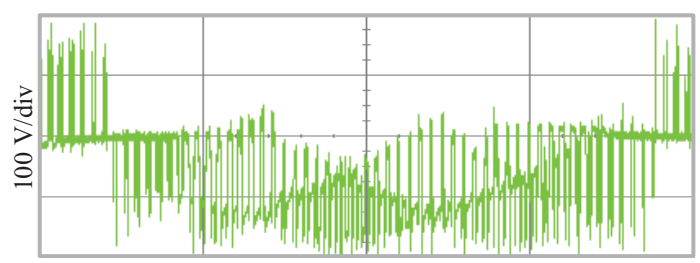

$19.2 \mathrm{~ms} / \mathrm{div}$

(c)

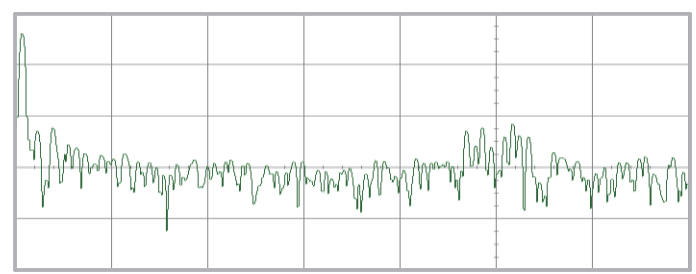

$1 \mathrm{kHz} / \mathrm{div}$

(d)

Fig. 5. Experimental waveforms under traditional modulation strategies (output phase voltage amplitude $70 \mathrm{~V} / 80 \mathrm{~Hz}$ ). (a) Three-phase output current. (b) u-phase input voltage and current. (c) Output line voltage PWM wave. (d) A output current spectrum $(20 \mathrm{~dB})$.

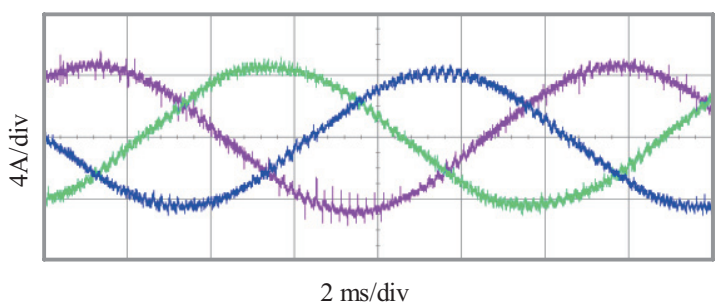

(a)

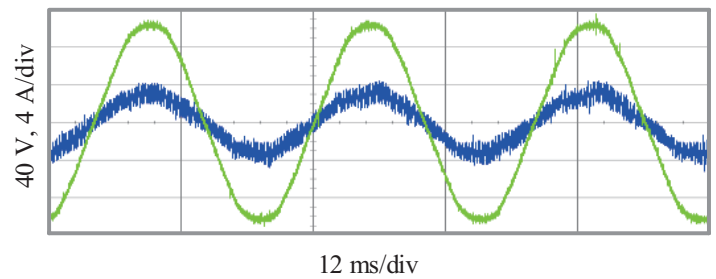

(b)

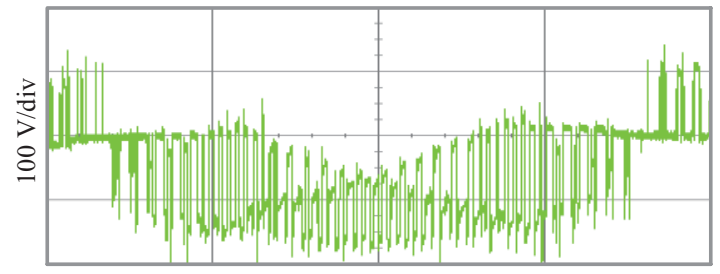

$19.2 \mathrm{~ms} / \mathrm{div}$

(c)

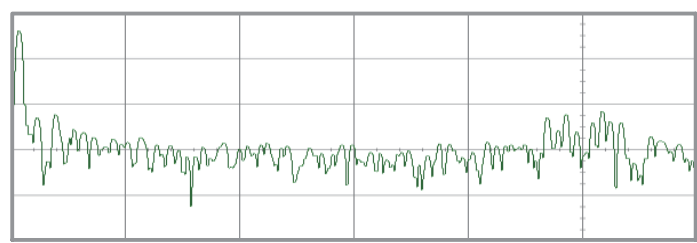

$1 \mathrm{kHz} / \mathrm{div}$

(d)

Fig. 6. Experimental waveform under new modulation strategy (output phase voltage amplitude $70 \mathrm{~V} / 80 \mathrm{~Hz}$ ). (a) Three-phase output current. (b) u-phase input voltage and current. (c) Output line voltage PWM wave. (d) A output current spectrum $(20 \mathrm{~dB})$. 


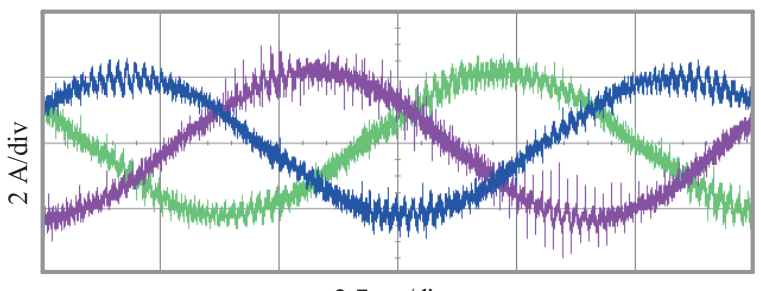

$2.7 \mathrm{~ms} / \mathrm{div}$

(a)

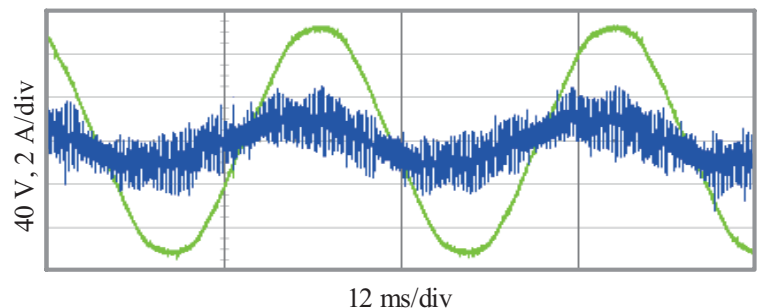

(b)

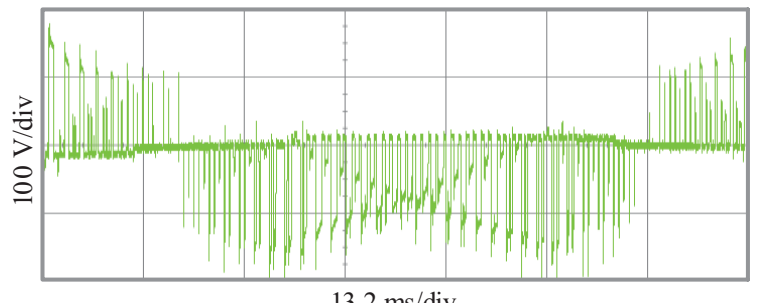

$13.2 \mathrm{~ms} / \mathrm{div}$

(c)

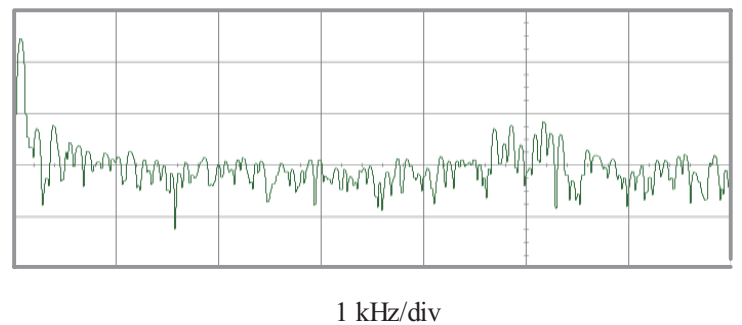

(d)

Fig. 7. Experimental waveform under new modulation strategy (output phase voltage amplitude $32 \mathrm{~V} / 80 \mathrm{~Hz}$ ). (a) Three-phase output current. (b) u-phase input voltage and current. (c) Output line voltage PWM wave. (d) A output current spectrum $(20 \mathrm{~dB})$.

From the experimental waveforms of Figs. 5 and 6. Under the traditional modulation strategy, when the output voltage frequency is $80 \mathrm{~Hz}$ and the amplitude is $70 \mathrm{~V}$, the THD value of the output current is $3.5 \%$. Under the new modulation strategy, the THD value of the output current is $2.4 \%$, it is lower than the THD measured under traditional modulation strategies, which is basically consistent with the simulation analysis. From the output line voltage PWM waveforms of Fig. 6(c) and Fig.7(c), it can be seen that when the reference output phase voltage amplitude is large, the maximum output line voltage phase is formed by three-input line voltage pulses, and no zero vector is used. When the reference output phase voltage amplitude is low, the three-output line voltages are formed by non-maximum two-input line voltage pulses and zero voltage. Consistent with the theoretical analysis mentioned above, the harmonic of output voltage is greatly suppressed.

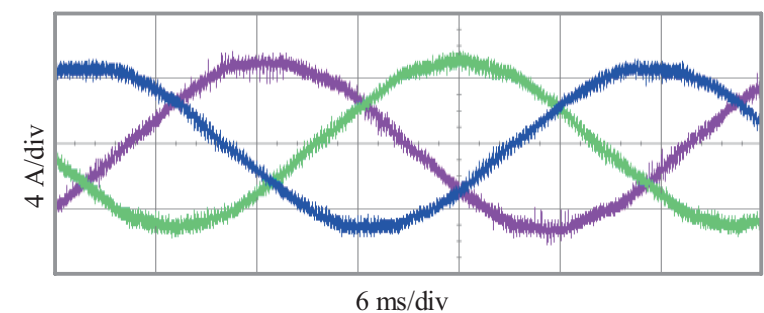

(a)

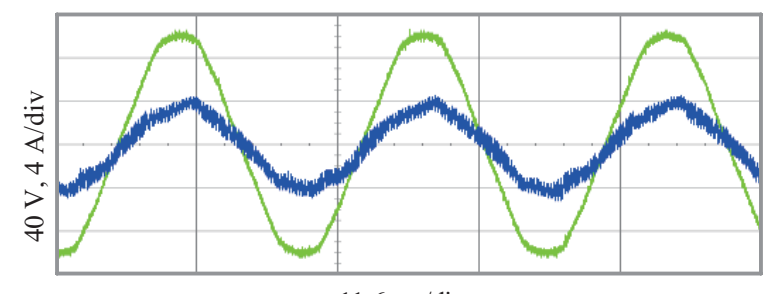

$11.6 \mathrm{~ms} / \mathrm{div}$

(b)

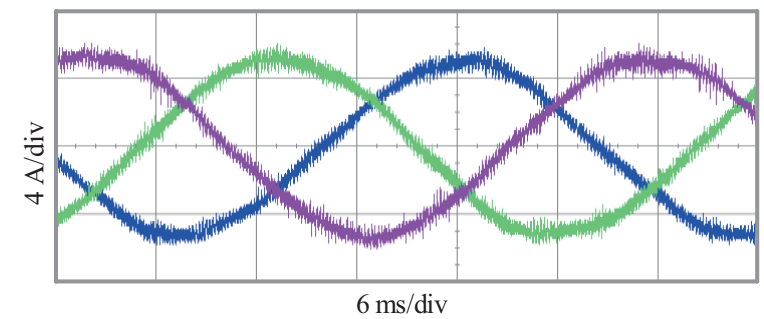

(c)

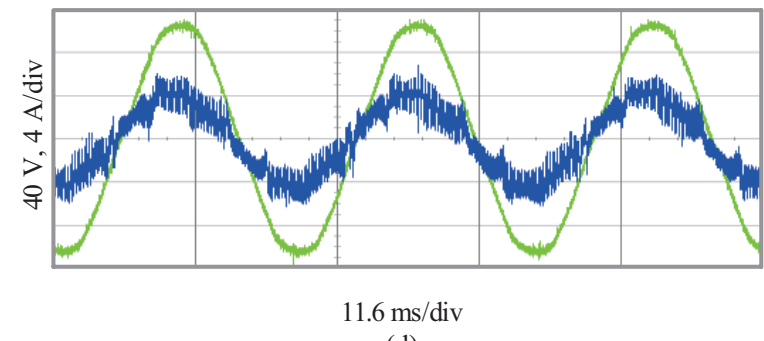

(d)

Fig. 8. Experimental waveform under new modulation strategy (output phase voltage amplitude $70 \mathrm{~V}, 30 \mathrm{~Hz})$. (a) Three-phase output current $\left(\varphi^{*}=0\right)$. (b) u-phase input voltage and current $\left(\varphi^{*}=0\right)$. (c)Three-phase output current $\left(\varphi^{*}=\right.$ $\pi / 6)$. (d)u-phase input voltage and current $\left(\varphi^{*}=\pi / 6\right)$.

Fig. 8 shows the relevant experimental waveforms when the desired output voltage amplitude is $70 \mathrm{~V} / 30 \mathrm{~Hz}$ and the input power factor angles $\varphi^{*}=0$ and $\varphi^{*}=\pi / 6$. From the input current and voltage waveforms in Fig. 8(b), it can be seen that due to the influence of the capacitance in the input filter, although the desired input power factor angle is $\varphi^{*}=0$, the input current lag voltage is about $24^{\circ}$. Fig. $8(\mathrm{~d})$ is a waveform of input current and voltage at the expected input power factor angle of $\varphi^{*}=$ $\pi / 6$. After offsetting the phase shift caused by the input filter, the phase difference of input current and voltage is about $6^{\circ}$.

Fig. 9 is an experimental waveform of common-mode voltage at load neutral point of traditional two-line voltage and proposed modulation strategy when the expected output voltage is $32 \mathrm{~V} / 30 \mathrm{~Hz}$ and the input power factor angle is $\varphi^{*}=0$. It can be seen from the waveform diagram that compared with the traditional two-line voltage strategy, the common mode 


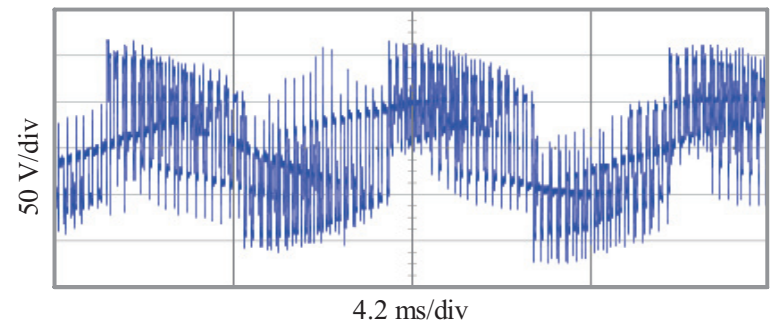

(a)

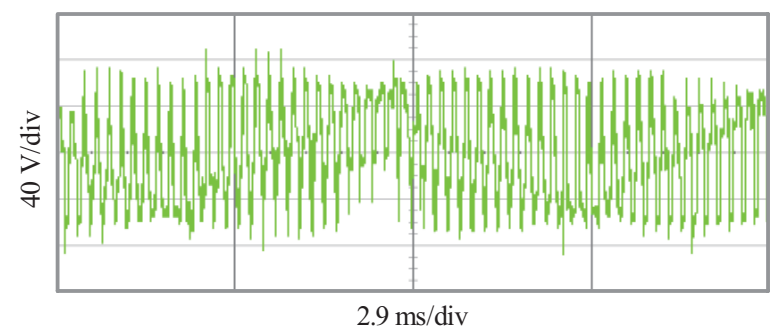

(b)

Fig. 9. Experimental waveforms of common mode voltage. (a) Common mode voltage under the traditional two-line voltage strategy. (b) Common mode voltage of the new modulation strategy.

voltage of the proposed modulation strategy load neutral point is suppressed. The reason is that the zero vector used in the new modulation strategy is the intermediate phase of the input voltage, while the zero vector used in the traditional two-line voltage strategy is the maximum value of the input voltage.

\section{CONCLUSIONS}

The simulation and experimental results show that the proposed modulation strategy improves the quality of output voltage under the same conditions compared with the traditional two-line voltage synthesis modulation strategy by changing the switching mode. Especially at low voltage output, the harmonic of output voltage is greatly suppressed, and the output performance of matrix converter is greatly improved. The zero vector used in the modulation process is the intermediate phase of input phase voltage, and the common mode voltage of load neutral point is suppressed. It can be seen that the new modulation strategy can achieve continuous control of high and low output voltage. While controlling the output voltage and input power factor, it reduces the output voltage harmonics and improves the output performance of matrix converter.

\section{REFERENCES}

[1] A. Ishiguro, T. Furuhashi, and S. Okuma, "A novel control method for forced commutated Cyclo-converters using matrix converter", IEEE Transactions on Industrid Electronucs, vol. 38, no. 3, pp. 166-172, 1991.

[2] E. Karaman, M. Farasat, and A.M. Trzynadlowski, "Comparative study of series and cascaded Z-source matrix converters", IEEE Transactions on Industrial Electronics, 2014, vol. 61, no. 10, pp. 5164-5173

[3] S. Ebrahimi, N. Amiri, H. Atighechi, et al, "Parametric average-value modeling of AC-AC matrix converters", Control and Modeling for Power Electronics, IEEE, 2016, pp. 1-7.

[4] S. Li, X. Yan, and Y. Zhong, "Improvement and optimization on hybrid two-stage Matrix coverter", in Proceedings of the CSU-EPSA, 2012, vol. 24, no. 3, pp. 145-149.

[5] Y. Wang, X. Chen, and D. Xu, "Research on closed-loop control method for Matrix Converter based on double line-to-line woltage synthesis", in Proceedings of the CSEE, vol. 22, no. 1, pp. 74-79, 2002.

[6] X. Ma, G. Tan, and X. Wang, "An improved two-line voltage synthesis control strategy of Matrix Converter", in Transactions of China Electrotechnical Society, 2009, vol. 24, no. 4, pp. 126-138.

[7] X. Wang, H. Lin, H. She, et al, "A research on space vector modulation strategy for matrix converter under abnormal inputvoltage conditions", IEEE Transactions on Industrial Electronics, vol. 59, no. 1, pp. 93-104, 2012.

[8] A. Zuckerberger and A. Alexandrovitz, "Matrix converter-theory and simulation", Eighteenth Convention of Electrical and Electronics Engineers in Israel, Mar 7-8 1995, pp. 4.5.5/1-4.5.5/5.

[9] H. Kim and S. K. Sul, "Analysis on output LC filters for PWM inverters", Power Electronics and Motion Control Conference, 2009. IPEMC '09. IEEE 6th International, May 17-20 2009, pp. 384-389

[10] G. Li, D. Zhang, Y. Xin, S. Jiang, W. Wang, and J. Du, "Design of MMC hardware-in-the-loop platform and controller test scheme," CPSS Transactions on Power Electronics and Applications, vol. 4, no. 2, pp. 143-151, June 2019.

[11] A. Iqbal, and S. Moinuddin "Comprehensive relationship between carrier-based PWM and space vector PWM in a five-phase VSI," IEEE Transactions on Power Electronics, vol. 24, no. 10, pp. 237923902009.

[12] D. Casadei, G. Serra, A. Tani, and L. Zarri, "Matrix converter modulation strategies: a new general approach based on space vector representation of the switch state," IEEE Transactions on Industrial. Electronics. vol. 49, no. 2, pp. 370-381, Apr. 2002.

[13] N. S. Choi, E. C. Lee, and K. S. Ahn, "A carrier-based medium vector PWM strategy for three-level inverters in transformer less photovoltaic systems," 2015 9th International Conference on Power Electronics and ECCE Asia (ICPE-ECCE Asia), Seoul, 2015, pp. $2752-2757$.

[14] T. D. Nguyen, D. Q. Phan, D. N. Dao, and H. H. Lee, "Carrier phase-shift PWM to reduce common-mode voltage for three-level t-type NPC inverters," Journal of Power Electronics, vol. 14, no. 6, pp.1197-1207, 2014.

[15] L. Kai, J. Zhao, W. Wu, M. Li, L. Ma and G. Zhang, "Performance analysis of zero common-mode voltage pulse-width modulation techniques for three-level neutral point clamped inverters," IET Power Electronics, vol. 9, no. 14, pp. 2654-2664, nov. 16, 2016.

[16] M. C. Cavalcanti, A. M. Farias, K. C. Oliveira, F. A. S. Neves, and J. L. Alfonzo, "Eliminating leakage currents in neutral point clamped inverters for photovoltaic systems," IEEE Transactions on Industrial. Electronics. vol. 59, no. 1, pp. 435-443, Jan. 2012.

[17] C. Wang and Y. Li, "Analysis and calculation of zero-sequence voltage considering neutral-point potential balancing in three-level NPC converters," IEEE Transactions on Industrial. Electronics. vol. 57, no. 7, pp. 2262-2271, July 2010.

[18] H. S. Jae, H. C. Chang, and S. H. Dong, "A new simplified space vector PWM method for three-level inverter," IEEE Transactionsons on Power Electronics, vol. 16, no. 4, pp. 545-550, 2001.

[19] H. Hara, E. Yamamoto, and J. K. Kang, "Improvement of output voltage control performance for low-speed operation of matrix converter." IEEE Transactions on Power Electronics, vol. 20, no. 6, pp. $1372-1378,2005$.

[20] J. Q. Han, "Fom PID to active disturbance rejection control," IEEE Transactions on Industrial Electronics, vol. 24, no. 11, pp. 120-126, 2009.

[21] H. J. Kim, H. D. Lee, and S. K. Sul, "A new PWM strategy for common mode voltage reduction in neutral-point-clamped invertersfed AC motor drives," IEEE Transactions on Industry Applications, vol. 37 , no. 6, pp. 1840-1845, Nov. 2001.

[22] J. R. Wang, Y. Zhong, and W. Song, "PR control for two-stage matrix converter excitation doubly fed wind generation system under unbalanced grid voltage conditions," Power \& Energy Engineering Conference IEEE, 2012.

[23] P. W. Wheeler, J. Clare, and L. Empringham, "Enhancement of matrix converter output waveform quality using minimized commutation times," IEEE Transactions on Industrial Electronics, vol. 51, no. 1, pp. 240-244, 2004.

[24] H. Wang, W. Wu, S. Zhang, Y. He, H. S.-H.Chung, and F. Blaabjerg, "A modified Aalborg inverter extracting maximum power from one PV array source," CPSS Transactions on Power Electronics and Applications, vol.4, no. 2, pp. 109-118, June 2019.

[25] dSPACE Real-time interface implementation guide (version4.2) [z],Paderborn: dSPACE GmbH, 2004. 


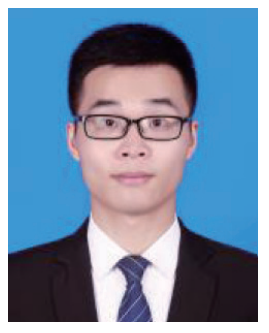

Yaguang Ma is a master student from the School of Electrical Engineering and Automation, Henan Polytechnic University. His research interests include power electronics systems and automatic control.

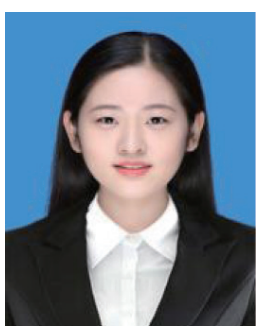

Peiru Li graduated from the School of Electrical Engineering and Automation, Henan Polytechnic University. She mainly engaged in power supply technology and electrical safety. Now, she is working at State Gird Henan Power Company Zhumadian Power Supply Company.

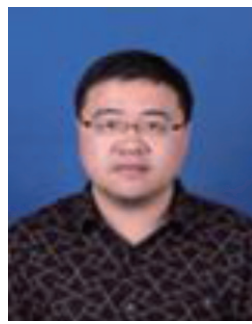

Xinghe Ma graduated from the School of Electrical Engineering and Automation, Henan Polytechnic University, for the degree of Bachelor and Master. He graduated from the School of Information and Electronics, China University of Mining, for the $\mathrm{Ph} . \mathrm{D}$. His research interests include power electronics and electric drive, automatic control, and intelligent information processing.

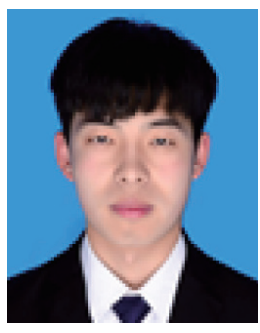

Xin Ren is a master student from the School of Electrical Engineering and Automation, Henan Polytechnic University. His research interests include power electronics systems and automatic control. 